\title{
Numerical investigation of a moisture wave-type vane separator
}

\author{
Ivan Kasatkin ${ }^{1, *}$, Mikle Egorov ${ }^{1}$ and Nikolay Rakov ${ }^{1}$ \\ ${ }^{1}$ Peter the Great St.Petersburg Polytechnic University, Russian Federation
}

\begin{abstract}
This study aims to determine the appropriate method for modeling separation processes in wavetype moisture vane separators and to analyze possible design improvements of the typical wave-type vane separator using numerical simulation methods. It discusses conditions of the secondary droplets generation phenomena. The applicability of the particle transport method for modeling the working process in wavetype vane separators is confirmed. The study explores the water droplets distribution pattern in the dispersed two-phase flow of a separator. A numerical study of the workflow in the typical steam-water separator with wave-type vanes was carried out. A dispersed two-phase flow in a separator is modelled as two separate flows. Dry saturated steam is considered as a continuous medium using the Euler method; the liquid phase is represented as a stream of water droplets described by the Lagrange method. Authors propose an option of modernization of the separator design applying three drainage channels on the path of small droplets.
\end{abstract}

\section{Introduction}

In nuclear power plant saturated steam turbines, separator-superheater provides acceptable humidity in the low-pressure section, which positively affects the reliability and efficiency of the turbine [1].

Mainly there are wave-type vane and high-speed centrifugal separators [2]. Typical design of a wave-type vane separator channel is shown in Figure 1 [3].

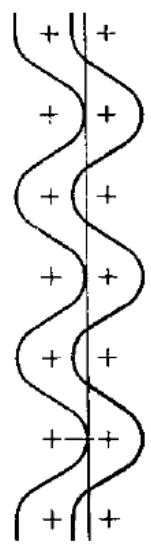

Fig. 1. A typical wave-type smooth vane separator channel.

The principle construction of a wave-type vane separator package is shown in Figure 2.

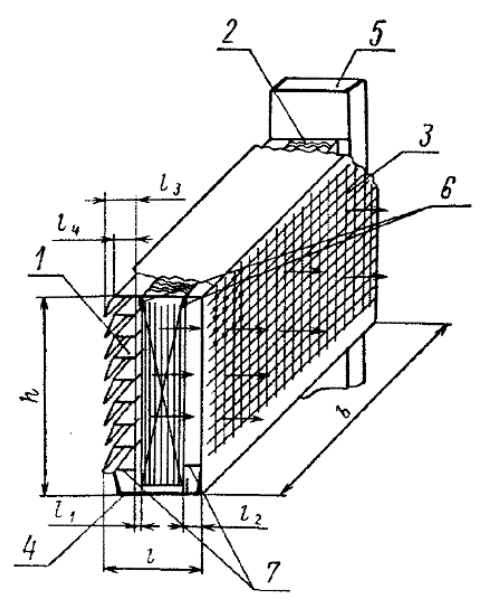

Fig. 2. Wave-type vane separator package principle construction. 1 - guide vanes; 2 - wave-type vanes; 3 perforated plate; 4-drain tank; 5 - drain channel; 6, 7 - slats.

The significant amount of moisture mostly presented by largest droplets settles on the walls of the guide vanes 1 installed in front of the vane separator packages 2. A perforated plate 3 is installed at the separator outlet for uniform steam flow. Fluid collected in drain tank 4 then comes in drain channel 5 . There are slats 6,7 at the bottom of the package to prevent the removal of the separated liquid.

Separation efficiency, humidity of the steam leaving the separator and separator pressure loss forms the primary performance of the moisture separator. Determination of these parameters from power plant tests gives the necessary information but these tests are of long duration and costly [4]. So using a numerical method for in-depth research of separation process is important for study and improving the equipment.

\footnotetext{
* Corresponding author: kasatkin_ivan@@mail.ru
} 
This study aims to determine the appropriate method for modeling separation processes in wave-type moisture vane separators and to analyze possible design improvements of the typical wave-type vane separator using numerical simulation methods.

\section{Methods}

To study the separation processes, a dispersed two-phase flow in a separator is modelled as two separate flows. Dry saturated steam is considered as a continuous medium using the Euler method; the liquid phase is represented as a stream of water droplets described by the Lagrange method

\subsection{Secondary droplets generation}

One of the reasons for incomplete drying of the steam in the separation part is the presence of small droplets in the steam generated during the separation process, which are likely to leave the separator evaluated with the steam flow.

This can occur as a result of detachment from larger droplets (2 in Figure 3), detachment from the liquid film on the vane due to collision with large drops or due to droplets dropping from the film due to interfacial shear stress (3 and 4 in Figure 3) [5].

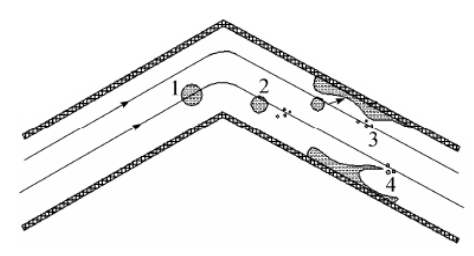

Fig. 3. Secondary droplets generation in a wave-type vane separator.

The destruction of droplets caused by the interaction with the continuous phase can be estimated based on the Weber number [6]:

$$
W e=\rho_{\mathrm{d}} \cdot u_{\mathrm{s}}^{2} \cdot \frac{D_{\mathrm{d}}}{\sigma},
$$

where $\rho_{\mathrm{d}}$ - water density, $u_{\mathrm{s}}-$ steam velocity, $D_{\mathrm{d}}-$ droplet diameter, $\sigma$ - surface tension of water.

The condition for tearing the film can be determined using the Weber number for the film [7]:

$$
W e_{\text {film }}=\rho_{\mathrm{s}} \cdot u_{\mathrm{s}}^{2} \cdot \frac{\delta}{\sigma}
$$

where $\rho_{\mathrm{s}}$ - steam density, $\delta$ - film thickness.

The critical value of the Weber number at which the destruction of the droplet occurs is 13 . The separation of droplets from the liquid film due to collision with large droplets occurs at We $>>1$ [8].

In the case under consideration, the flow rates are small, and for the largest drops $(50 \mu \mathrm{m})$, the Weber number is $\mathrm{We}=1.2$. Therefore, in this calculation, the secondary droplets generation is not considered.

\subsection{Particle transport model}

The simulation is carried out in the Ansys CFX software with the Particle Transport Fluid model to describe the movement of water droplets. The steady-state condition is considered. The boundary conditions at the inlet of the separator are:

- the velocities of steam and water droplets at the inlet to the separator are equal to $1.4 \mathrm{~m} / \mathrm{s}$;

- the mass flow rate of the droplets is selected so that the humidity at the inlet of the separator is $10 \%$;

- the initial position of the droplets is random, uniformly distributed over the entrance surface.

Since the height of the separator vanes is large, a two-dimensional problem is considered in a horizontal plane.

The particle transport model makes it possible to track the trajectory of water droplets in a steam flow. The separation of water droplets occurs due to their deposition on the wall, therefore, this model assumes that when a droplet touches the wall of the vane separator, it remains on it and flows down into the drain tank.

The calculation using this model is only possible with a sufficiently large number of particles and with small values of volumetric water content [9].

Given the small size of the droplets $(<150 \mu \mathrm{m})$ compared with the distance between the walls of the separator $(10 \mathrm{~mm})$, the number of droplets will be quite large. Given the relatively low steam humidity in the separator $(<16 \%)$, the volumetric water content will be quite low.

\subsection{Drag force}

The study accepts that only drag force affects droplets. The work takes the spherical shape of the particles, therefore, the drag force is determined as follows [10]:

$$
\overrightarrow{F_{\text {drag }}}=C_{\mathrm{D}} \cdot \frac{\pi \cdot D_{\mathrm{d}}^{2}}{8} \cdot \rho_{\mathrm{s}} \cdot\left|\vec{u}-\overrightarrow{u_{\mathrm{d}}}\right| \cdot\left(\vec{u}-\overrightarrow{u_{\mathrm{d}}}\right),
$$

where: $C_{\mathrm{D}}-$ drag resistance coefficient, $\vec{u}-$ flow velocity, $\overrightarrow{u_{d}}-$ droplet velocity.

The drag resistance coefficient is defined by SchillerNauman [11]:

$$
C_{D}=\frac{24}{R e} \cdot\left(1+0,15 \cdot R e^{0,687}\right),
$$

\subsection{Droplets size distribution}

The droplets size distribution is greatly important in calculating the separation efficiency.

The volumetric distribution of droplets by size in the dispersed-annular flow of the wave-type vane separator is [12]: 


$$
\begin{aligned}
& f_{v}\left(d_{\mathrm{d}}\right)=\frac{\delta \cdot d_{\mathrm{d} \max }}{\sqrt{\pi} \cdot d_{\mathrm{d}} \cdot\left(d_{\mathrm{d} \max }-d_{\mathrm{d}}\right)} \times \\
& \times \exp \left(-\delta^{2} \cdot\left(\ln \left(\frac{d_{\mathrm{d}}}{a \cdot\left(d_{\mathrm{d} \max }-d_{\mathrm{d}}\right)}\right)^{2}\right)\right)
\end{aligned}
$$

where $\quad a=\frac{d_{\mathrm{d} v, \mu}}{d_{\mathrm{d} \max -d_{\mathrm{d} v, \mu}}}, \quad d_{\mathrm{d} v, \mu}$ - average median diameter, $\delta$ - distribution parameter.

The distribution parameter is related to the confidence interval for the results obtained in the experiment. The values of $d_{\mathrm{d} v, \mu}$ and $d_{\mathrm{d} \text { max }}$ depend on the experimental parameters (channel size, medium properties, etc.) and can be found from expressions [13]:

$$
\frac{d_{\mathrm{d} \max }}{d}=0,031 \cdot \frac{R e_{\mathrm{d}}^{0,67}}{W e_{\mathrm{d}}} \cdot\left(\frac{\rho_{\mathrm{d}}}{\rho_{\mathrm{s}}}\right)^{0,33} \cdot\left(\frac{\mu_{\mathrm{s}}}{\mu_{\mathrm{d}}}\right)^{0,67},
$$

where $R e_{\mathrm{\kappa}}-$ droplets Reynolds number,

$$
R e_{\mathrm{d}}=\frac{\rho_{\mathrm{d}} \cdot u_{\mathrm{d}} \cdot D_{\mathrm{H}}}{\mu_{\mathrm{d}}},
$$

$\mu_{\mathrm{s}}, \mu_{\mathrm{d}}-$ cinematic viscosity of steam and droplets, respectively, $D_{\mathrm{H}}-$ hydraulic diameter of vane package cross-section.

The volumetric distribution of droplets in studied case is shown in Figure 4.

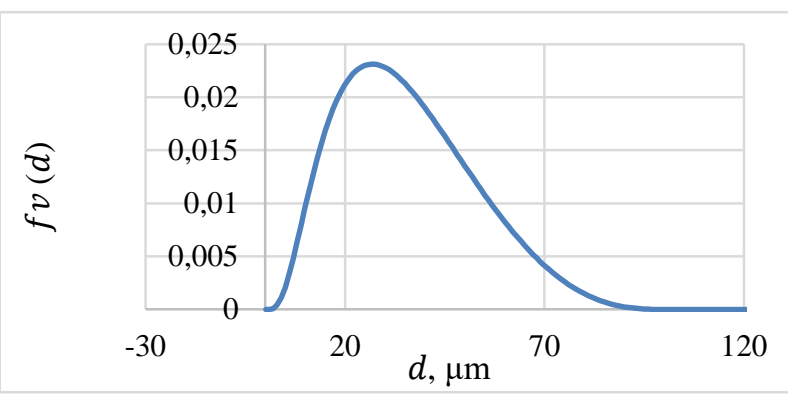

Fig. 3. The volumetric distribution of droplets in studied vane separator.

\subsection{Assumptions}

The study considers several assumptions:

1. Due to the small droplet size and low Weber number $\mathrm{We} \leq 1$, the interaction of the droplets with the film and the secondary droplets generation in the separator are not considered in the calculation.

2. The average distance between particles is large enough [14]. Thus, the droplets: do not constrain the movement of each other during mutual displacement; do not collide; do not coagulate; are not destroyed.

3. In view of the small difference in the velocities of the liquid and continuous phases, the velocities of the droplets and vapor at the inlet are assumed to be equal in a first approximation [15].

\section{Results}

Figure 4 shows the trajectories of water droplets obtained by numerical simulation and the distribution by the diameter of the droplets in the calculated area of the separator.

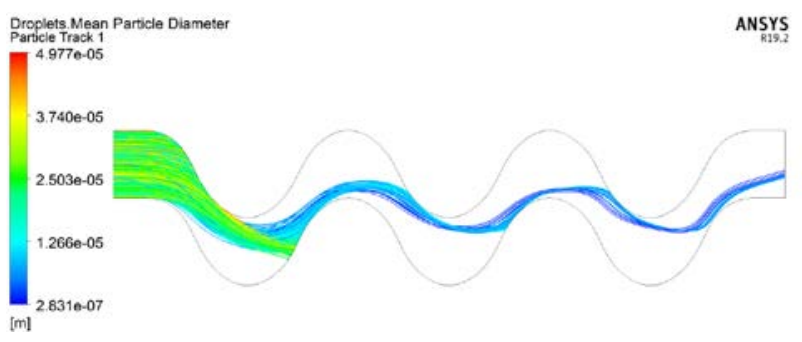

Fig. 4. Trajectories and distribution of droplet diameters over the computational domain in the separator.

The separation efficiency is:

$$
\eta=\left(1-\frac{G_{\mathrm{d}}^{\text {outlet }}}{G_{\mathrm{d}}^{\text {inlet }}}\right) \cdot 100 \%=88.2 \%
$$

According to the results of numerical simulation, the pressure loss in the wave-type vane separator is $46.0 \mathrm{~Pa}$.

Then, an analytical calculation of the pressure loss is performed [16].

The separator pressure losses $\Delta p_{\text {sep }}$ :

$$
\Delta p_{\text {sep }}=\xi_{\text {sep }} \cdot \frac{u_{\mathrm{s}}^{2}}{2} \cdot \rho_{\mathrm{s}}=44.8 \mathrm{~Pa} \text {, }
$$

where $\xi_{\text {sep }}$ - hydraulic resistance coefficient of the separator.

The relative difference between the pressure loss values obtained analytically and using numerical simulation is $2.6 \%$.

\section{Discussion}

Based on the analysis of the droplet trajectories, it is proposed to make a change in the design of the separator and add drainage channels on the path of small droplets starting from the third turn of the channel [17]. The simulation results of the proposed design are presented in Figure 5.

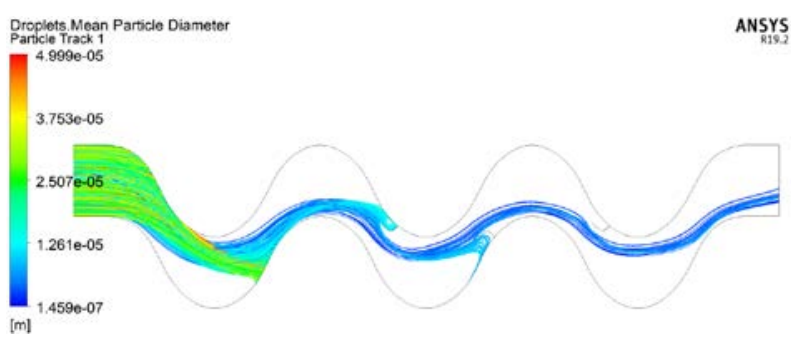

Fig. 5. Trajectories distribution of droplet diameters over the computational domain in the separator with drainage channels. 
According to the results of numerical simulation, the efficiency of the separator with drainage channels is:

$$
\eta=\left(1-\frac{G_{\mathrm{d}}^{\text {outlet }}}{G_{\mathrm{d}}^{\text {inlet }}}\right) \cdot 100 \%=91.2 \%,
$$

The pressure loss in the separator with drainage channels is $62.4 \mathrm{~Pa}$.

When adding the drainage channels, pressure loss increased by $35.5 \%$, separation efficiency increased by $2 \%$. Considering the fact that the ratio of the pressure loss in the vane separator to the entire separatorsuperheater is $0.2 \%$, such an increase in pressure loss can be considered insignificant.

\section{Conclusions}

1. Using numerical simulation with particle transport model, the trajectories of water droplets and the distribution of droplets in diameter, velocity field and steam pressure field are obtained. The value of the vapor pressure loss determined using numerical simulation coincides with the value calculated analytically with an accuracy of $2.6 \%$.

2. Possible options for improving the design of the separator are to reduce the length of the channel and add drainage channels along the path of the trajectory of small drops.

3. Adding three drainage channels on the path of small droplets, the thickness of which is $25 \%$ of the distance between the vanes of the separator, allows to increase the efficiency of the separator by $2 \%$.

\section{References}

1. A. Ulasen, A. Kalyutik, and A. Blagoveshchenskii, MATEC Web Conf. 245, (2018)

2. L. Liu and B. Bai, Nucl. Eng. Des. 298, 229 (2016)

3. V. Legkostupova and A. Sudakov, Therm. Eng. 62, (2015)

4. M. Le, J. Kim, J. Kim, H. S. Do, and J. Lee, Nondestruct. Test. Eval. 33, 35 (2018)

5. D. B. Hann, A. V. Cherdantsev, A. Mitchell, I. N. McCarthy, B. N. Hewakandamby, and K. Simmons, Exp. Fluids 57, 1 (2016)

6. J. R. Thome and A. Cioncolini, Entrained Liquid Fraction in Annular Two-Phase Flow (2015)

7. X. H. Tan, J. Y. Liu, X. P. Li, G. D. Zhang, and C. Tang, Math. Probl. Eng. 2013, (2013)

8. S. H. Pham, Z. Kawara, T. Yokomine, and T. Kunugi, Int. J. Multiph. Flow 70, 35 (2015)

9. F. Zhao, C. Zhao, and H. Bo, Ann. Nucl. Energy 111, 176 (2018)

10. M. Petrichenko, V. Sergeev, E. Kotov, D. Nemova, and D. Tarasova, in Int. Sci. Conf. Energy Manag. Munic. Facil. Sustain. Energy Technol. EMMFT 2018, edited by V. Murgul and M. Pasetti (Springer International Publishing, Cham, 2019), pp. 839-848

11. A. S. Stabnikov and A. V. Garbaruk, J. Phys. Conf. Ser. 1135, (2018)
12. N. D. Agafonova and I. L. Paramonova, J. Eng. Phys. Thermophys. 89, 840 (2016)

13. J. Steimes and P. Hendrick, Int. J. Multiph. Flow 93, 84 (2017)

14. J. Li, S. Huang, and X. Wang, Chinese J. Chem. Eng. 15, 492 (2007)

15. Y. Liu and Z. Qu, Energy Procedia 105, 1501 (2017)

16. H. W. Dries and A. C. Hoffmann, AIChE J. 65, 1 (2019)

17. F. Kavousi, Y. Behjat, and S. Shahhosseini, Chem. Eng. Res. Des. 91, 1212 (2013) 